\title{
Agricultural Stress Monitoring using Remote Sensing Data
}

\author{
Kavita V. Bhosle, Vijaya Musande
}

\begin{abstract}
This study consist of experiments on Hyperspectral remote sensing data for monitoring field stress using remote sensing tools. We have segmented Hyperspectral image and then calculated stress level using ENVI tool. EO-I hyperspectral remote sensing data from hyperion space born sensor has been used as the key input. QUACK (Quick Atmospheric Correction) algorithm has been used for atmospheric correction of hyperspectral data. EO-1, hyperion sensors data It has been observed that stress level depends on chlorophyll contents of a leaf. It has been observed that green field is with less stress and rock where no chlorophyll contents have most stress. We have also shown stress level in the scale of 1 to 9 .
\end{abstract}

Keywords: Atmospheric correction, Hyperspectral remote sensing data, Field Stress.

\section{INTRODUCTION}

Remote sensing data has great useful data which can be used for different field applications. Popular application of remote sensing is land cover land use. Role of vegetation analysis is more useful for sustainable development [1]. Crop identification and area estimation is one of the applications [2][3]. Land use land cover classification is very useful and used by many researchers [4]. Peoples have used many methods for classification. Indices are very useful parameter for classification. There are mono spectral, multispectral and hyperspectral remote sensing data available which has different significance and has different number of bands. Monospectral has single band, multispectral has many and non contiguous bands. Hyperspectral has thousands of bands and all are contiguous. Large number of information is available in hyperspectral data. Thenkabail found best wavebands for vegetation analysis to assess the vegetation and agricultural crop classification [5]. Vegetation Change may be Detected using remote sensing data and vegetation analysis method [6]. Vegetation identification using hyperspectral remote sensing data has been implemented by Erin et al.[7]. Modeling and vegetation analysis using remote sensing data for has been used by Goodchild [8].

Revised Manuscript Received on January 15, 2020.

* Correspondence Author

Kavita Bhosle*, Department of CSE, Maharashtra Institute of Technology, Aurangabad, Maharashtra, India.

Dr. Vijaya Musande, Department of CSE, MGM's Jawaharlal Nehru Engineering College, Aurangabad, Maharashtra, India.

(C) The Authors. Published by Blue Eyes Intelligence Engineering and Sciences Publication (BEIESP). This is an open access article under the CC BY-NC-ND license (http://creativecommons.org/licenses/by-nc-nd/4.0/)

\section{METHODS AND DATASET}

\section{A. Area under Study}

We have chosen area of Aurangabad region, MH state of India for study land cover stress level. In the last week of Dec 2015, we have made survey and collected ground truth. EO1sensor, which is Hyperion, was chosen to acquire required images of required time stamp. Table-I consist of the detail location of area under study.

Table- I: Details of area under study (latitude, longitude)

\begin{tabular}{|c|c|}
\hline Left upper corner & $20.31,75.4$ \\
\hline Right upper corner & $20.29,75.47$ \\
\hline Left lower corner & $19.37,75.26$ \\
\hline Right lower corner & $19.39,75.18$ \\
\hline
\end{tabular}

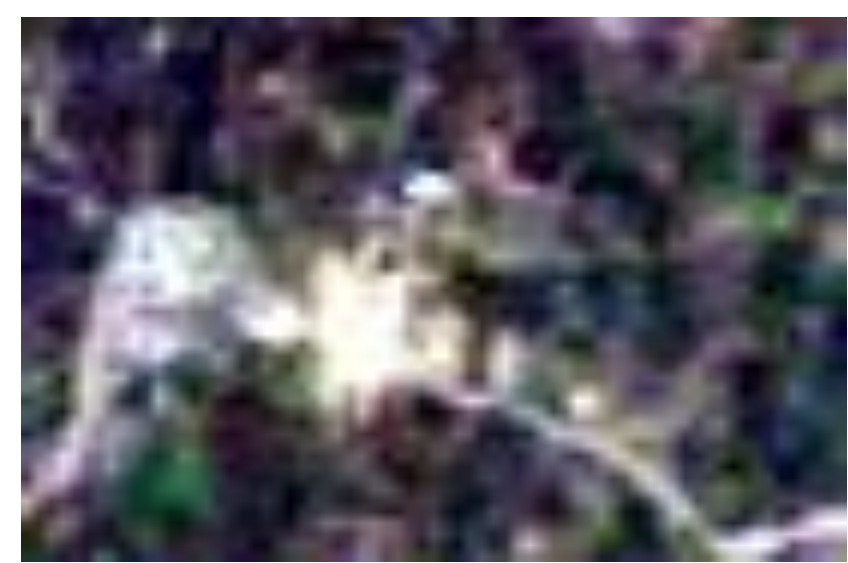

Fig. 1. Segment input image of region of interest

\section{B. Preprocessing}

The Input image is of 3381 (49 x 69) pixels. The main advantage of using hyperspectral data is that it has thousands of contiguous bands which contain lots of meaningful information. The disadvantage is that it required preprocessing due to atmospheric effect. QUACK (Quick Atmospheric Correction) algorithm has been used for atmospheric correction of hyperspectral data. EO-1, hyperion sensors data of dated 24th Dec 2015 has been used for experimentation purpose. This Hyperspectral data consist of 242 bands. We have obtained 155 bands by applying QUACK and by removing bad bands [9][10].

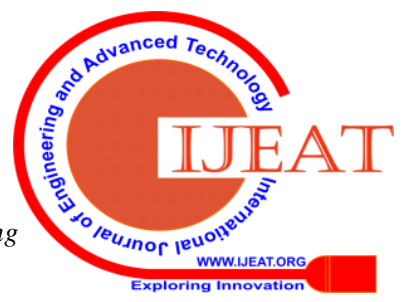




\section{Agricultural Stress Monitoring using Remote Sensing Data}

\section{Segmentation}

Segmentation is necessary to evaluate and analyse methods and techniques effectively [11]. After preprocessing or atmospheric correction of hyperspectral image, we have segmented this image. We have ground truth of this segment or Region of interest (ROI) is of Waregaon village has been collected which has been shown in study area. We have extracted image that we want to analysis using segmentation feature of ENVI. We have provided this segment for vegetation analysis. Fig 1 shows segment of hyperspectral image acquired which has been used for vegetation analysis purpose.

\section{Vegetation Analysis}

Vegetation analysis is used to find stress on different plants of fields. ENVI tool has been used for vegetation analysis. Physical property of each material is different. When sun light incidents on any material reflection value is different and it depends on properties of material.

This principle has been used to identify material. Remote sensing uses the electromagnetic spectrum. It gives us the electromagnetic energy reflected or emitted by the material which is available on earth's surface. Remote sensing acquired the information and data about an process or object without establishing physical contact with the process or object. Due to different level of chlorophyll contents present in each leaf, stress level can be decided. If leaves are more stresses then chlorophyll contents are less. If material are rock, stone, road then there is no chlorophyll contents. In this case stress level is more. This can be used to identify either stresses plants or fields as well as land cover material.

ENVI tool gives us 9 levels of stress. Purple color or level 1 indicate less stress and red color or level 9 indicate more stress as shown in figure.

Spectral Vegetation Analysis method has been used for finding agricultural Stress. Fig 2 shows flow of method used for analysis.

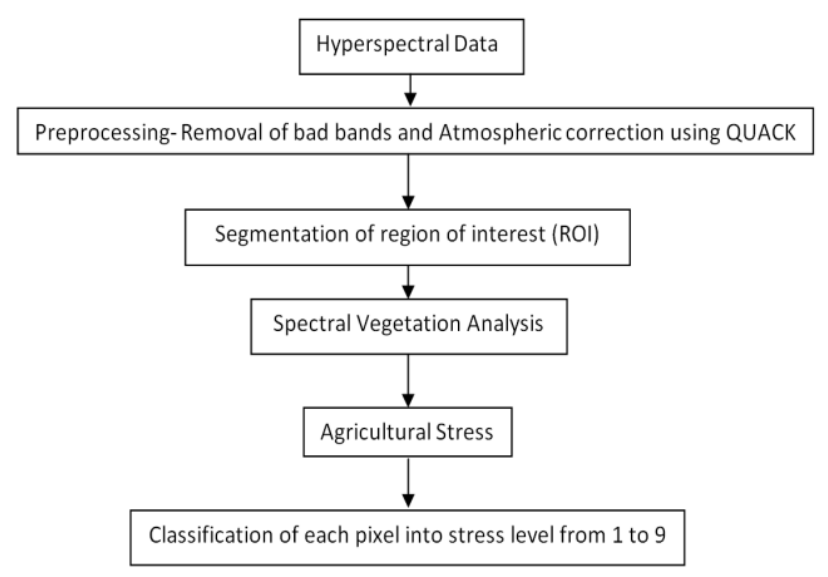

Fig. 2. Flow of methods used for vegetation analysis

\section{RESULT AND DISCUSSIONS}

It has been observed that result of vegetation analysis has given clear picture of classification of rock area from green vegetation area. Rock area has been shown by red color in figure. It indicates that no green area or no chlorophyll contents available so shown level 9. For other green area stressed level shown from 1 to 8 depending upon greenness of plants. Out of 3381 pixel 2558 pixels are correctly identified therefore accuracy is $75.65 \%$.
After analysis of vegetation stress fig 3 shows stress level of each pixel varying from 0 to 9 . Level 0 indicates that pixel not classified. Level 1 indicates that pixel under less stress more chlorophyll contents. Level 9 indicated that no vegetation so had more stress. Fig 4 shows stress level using color code. Purple with less stress, Red with more stress and black with not classified pixel shown in figure.

Fig 5 shows graphical representation of number of pixel in each stress level and fig 6 shows percentage of pixel in each stress level for segmented region of interest.

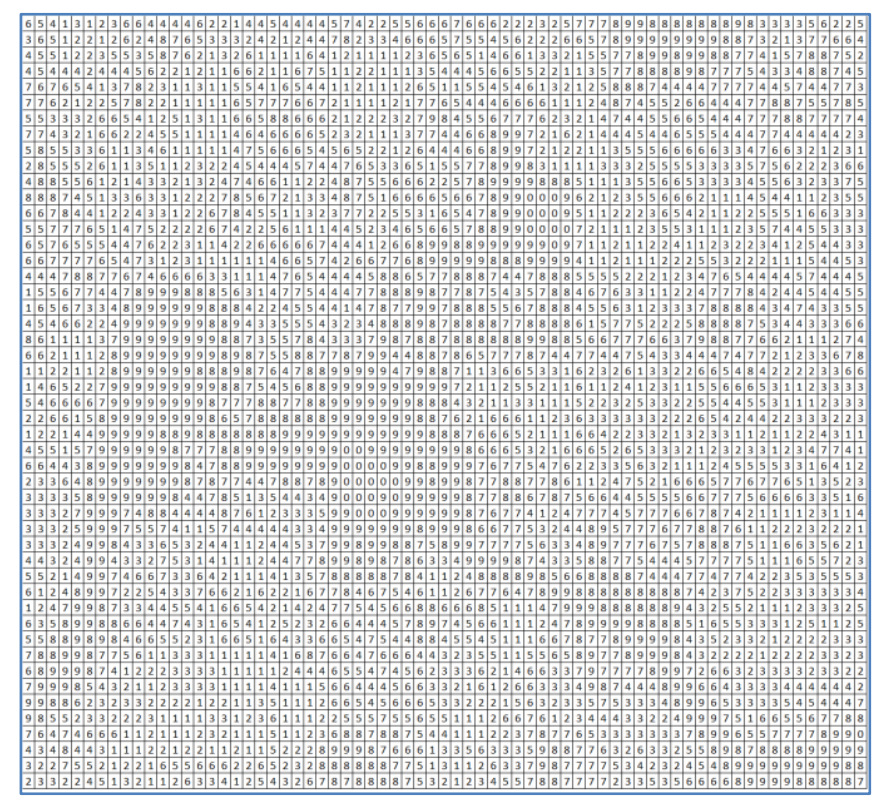

Fig. 3. Stress level of each pixel matrix

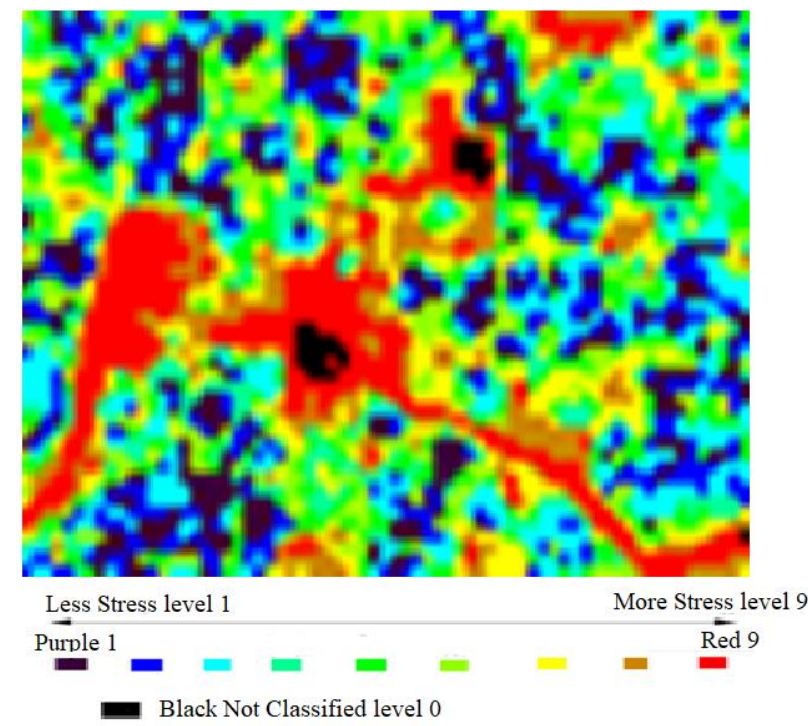

Fig. 4. Stress level of each pixel in the segment

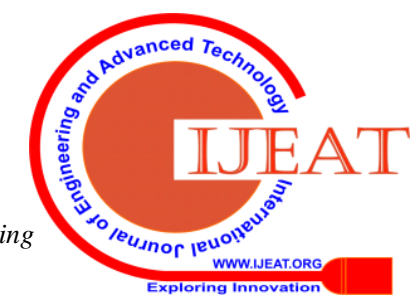




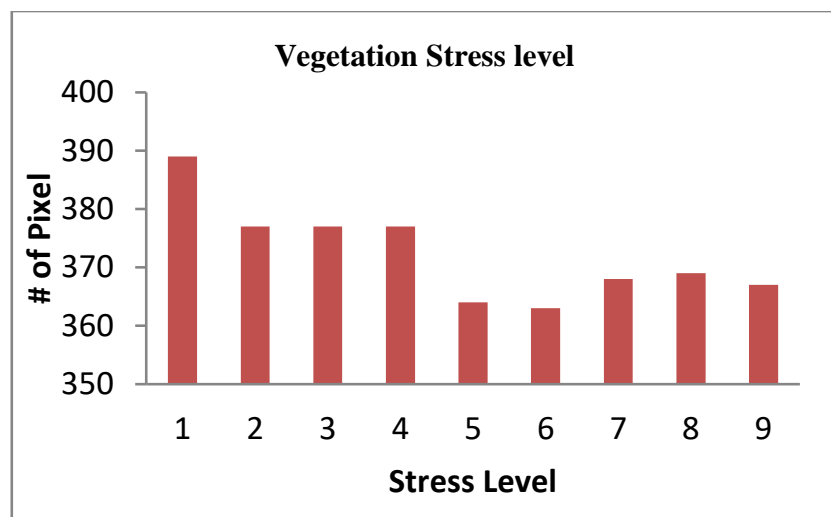

Fig. 5. Number of pixel for each vegetation stress level

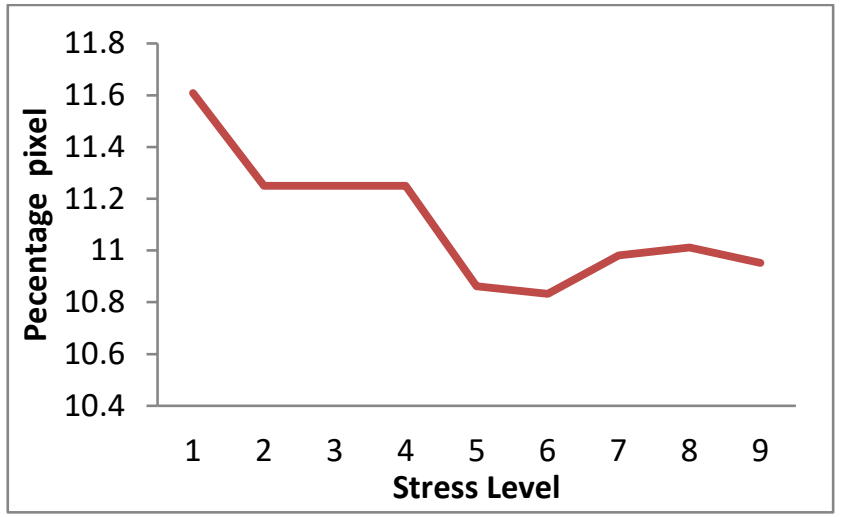

Fig. 6. Percentage of pixel for each vegetation stress level

\section{CONCLUSION}

Hyperspectral data is very useful as it contains contiguous many numbers of bands. Only atmospheric correction is required to Hyperspectral data as it is more affected by atmospheric noise. So we have been used QUACK (Quick Atmospheric Correction) method for atmospheric correction. In this paper, Vegetation stress level has been calculated using ENVI tool. Spectral Vegetation Analysis method has been used for finding agricultural stress level. It has been observed that $75.65 \%$ accuracy obtained for pixel wise stress calculation. Agricultural stress has been classified into scale of 9 . Level 1 for less stressed pixel and 9 for more stressed pixel.

Remote sensing images can be used for land cover land use classification and for finding agricultural stress and for vegetation analysis.

\section{REFERENCES}

1. P. Almeida,A. Altobelli,L. D'Aietti,E. Feoli,P. Ganis,F. Giordano, The role of vegetation analysis by remote sensing and GIS technology for planning sustainable development: A case study for the Santos estuary drainage basin (Brazil).

2. V Musande, Anil Kumar, Karbhari Kale, "Cotton Crop Discrimination Using Fuzzy Classification Approach”, 40(4):589-597, Springer J Indian Soc Remote Sens.

3. Vijaya Musande, Anil Kumar, P.S. Roy, Karbhari Kale, "Evaluation of fuzzy-based classifiers for cotton crop identification" , 28:3, 243-257, Geocarto International, Taylor and Francis.

4. Bin Zhang, Yueyan Liu, Zuyu Zhang, Yonglin Shen, 2017, "Land use and land cover classification for rural residential areas in China using soft-probability cascading of multifeatures". Journal of Applied Remote Sensing, Vol. 11(4)

5. Prasad S.Thenkabail, Eden A.Enclona, Mark S.Ashton, BaukeVan Der Meer, Accuracy assessments of hyperspectral waveband performance

for vegetation analysis applications, Remote Sensing of Environment, Volume 91, Issues 3-4, 30 June 2004, Pages 354-376

6. G. MeeraGandhi, S.Parthiban, NagarajThummalu, A.Christy, Ndvi: Vegetation Change Detection Using Remote Sensing and Gis - A Case Study of Vellore District, Procedia Computer Science Volume 57, 2015, Pages 1199-1210

7. Erin L.Hestir, ShrutiKhanna, Margaret E.Andrew, Maria J.Santos, Joshua H.Viers, Jonathan A.Greenberg, Sepalika S.Rajapakse, Susan L.Ustin, Identification of invasive vegetation using hyperspectral remote sensing in the California Delta ecosystem, Remote Sensing of Environment

Volume 112, Issue 11, 15 November 2008, Pages 4034-4047

8. Michael F. Goodchild, Integrating GIS and remote sensing for vegetation analysis and modeling: methodological issues, Journal of Vegetation Science, October 1994, https://doi.org/10.2307/3235878

9. Lawrence S. Bernstein, Xuemin Jin, Brian Gregor, and Steven M. Adler-Golden "Quick atmospheric correction code: algorithm description and recent upgrades," Optical Engineering 51(11), 111719 (31 July 2012). https://doi.org/10.1117/1.OE.51.11.111719

10. Yunkai GUO, Fan ZENG, ATMOSPHERIC CORRECTION COMPARISON OF SPOT-5 IMAGE BASED ON MODEL FLAASH AND MODEL QUAC International Archives of the Photogrammetry, Remote Sensing and Spatial Information Sciences, Volume XXXIX-B7, 2012 XXII ISPRS Congress, 25 August - 01 September 2012, Melbourne, Australia

11. Chengming Zhang, Jiping Liu, Fan Yu, Shujing Wan, Yingjuan Han, Jing Wang, and Gang Wang, "Segmentation model based on convolutional neural networks for extracting vegetation from Gaofen-2 images", J. Appl. Remote Sens. 12(4), 042804 (2018), doi: 10.1117/1.JRS.12.042804

\section{AUTHORS PROFILE}
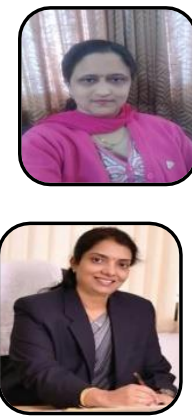

Bhosle Kavita, has completed Bachelor of Engineering and Master of Engineering in computer Science and Engineering. She is pursuing $\mathrm{PhD}$. She is Associate Professor in CSE department of MIT, Aurangabad, MH, India. Remote sensing, artificial neural network and deep learning are her research areas.

Dr. Musande Vijaya, has completed $\mathrm{PhD}$ in Computer Science and Engineering from Dr. Babasaheb Ambedka Marathwada University, Aurangabad, MH, India. She is Professor in CSE Department, MGM's Jawaharlal Nehru Engineering College, Aurangabad, $\mathrm{MH}$, India. Machine learning, deep learning Remote sensing are her research areas. 